conventional $5 \%$ level of statistical significance. The reinfarction rate for males in the "high-dosage" group, however, is significantly less than in the "low-dosage "group. The evidence, both clinical and electrocardiographic, on which the diagnosis of reinfarction was made was assessed, admittedly retrospectively, by a group of observers who did not know to which group the patients belonged. The results of this independent assessment indicate that the accuracy of the diagnosis of reinfarction by the participating clinicians was similar in the two groups. Consequently the excess incidence of reinfarction in the "low-dosage" group was unlikely to be due to observer bias in favour of anticongulant therapy. Though the number of women included in the trial was small, there is again no evidence that females obtain any benefit from anticoagulant therapy after myocardial infarction.

The second question which must be answered is : "If longterm anticoagulant therapy is of value, how long does the benefit last ?" There were indications in the original report to the M.R.C. and in some of the other trials that the beneficial effects are greatest in the months immediately after an infarction, and that as time goes on the differences between the two groups for both mortality and the incidence of reinfarction become less evident. This naturally raises the question whether the observed early benefit might be more apparent than real and due to the cessation of short-term anticoagulant treatment in patients who had received the drugs during the acute phase of their illness. When this was stopped on their entering the trial they might have suffered thrombosis from a "rebound" effect. ${ }^{3}$ This possibility has been considered in the present report, and it concludes that this is not the cause of the higher death rate and the greater incidence of reinfarction in the "low-dosage" group, as these continue for some months after the acute phase of the illness.

In the final stage of the trial, after all the patients had been followed up for at least two years, the original " high-dosage " group was divided into two groups : half of the patients had their anticoagulants reduced to the "low-dosage" level over a period of three weeks and the other half continued to receive a therapeutic dosage of phenindione. The patients in these two new groups were paired, and over the next thirty months the results were followed by the procedure of sequential analysis. The number of patients in this phase of the trial was small, but the results do not suggest that any great benefits accrue from the continuation of anticoagulant therapy beyond two years, or that there is any particular risk associated with the withdrawal of anticoagulants after two to three years of treatment. In recent weeks the preliminary report of a further trial in the United States has also been published. ${ }^{4}$ This is a well-designed study and has now been running for seven years, with 196 patients included. To date the results do not show any difference in either mortality or morbidity between patients receiving and not receiving anticoagulants. The patients in this trial have not been subdivided by age or sex.

From all this it is perhaps reasonable to conclude that a male who has recovered from the immediate effects of an acute myocardial infarction is likely to have a better prognosis over the subsequent one to two years if he is given anticoagulant therapy ; during this period his chances of survival

1 Report to Medical Research Council, Brit. med. f., 1959, 1, 803.

McMichael, J., ibid., 1959, 1, 970.

Ibid., 1962, 2, 659 .

- Seaman, A. J., Griswold, H. E., Reaume, R. B., and Ritzman, L. W., f. Amer. med. Ass., 1964, 189, 183.

- Lovell, R. R. H., Brit. med. F., 1964, 2, 465. are probably better, and, especially if he is less than 55 years old, his chances of escaping a further infarction are appreciably better if he is given this form of treatment. But beyond this initial period the prognosis is apparently unaffected by the continuation of anticoagulant treatment, and it may then be withdrawn without untoward effects. But in spite of all the work that has been done the situation is still not clear and it is impossible to be dogmatic about anticoagulant therapy for these patients. The general impression is that anticoagulants confer a marginal benefit. If they do, then, as R. R. H. Lovell ${ }^{5}$ has recently pointed out in this journal, a trial containing a much larger number of patients than even the sum of those in all the trials conducted so far will be required to demonstrate this benefit convincingly. Moreover, if the advantages are slight the potential dangers of anticoagulant therapy assume greater importance. While they are not negligible, the experience of all the trials does not suggest that they are prohibitive. But it is worth noting that these results apply only to hospitals where the physicians are interested and experienced in the use of anticoagulants, and that if the treatment were more widely adopted the hazards might be greater. If one considers the problem in more detail, and attempts to answer the question, "Which patient, if any, will benefit from anticoagulant therapy ?" the answer is clearly the younger male patient, and that he should be treated for one to two years after infarction. In the light of our present knowledge it may be considered reasonable to treat older male patients also for a similar period, but there seems to be little justification for giving women long-term anticoagulant therapy.

\section{Replacing Limbs}

Though successful repair of incomplete amputation of limbs has been undertaken on many occasions ${ }^{1}$ successful replacement of limbs that have been totally severed from the body is still rare. This apparent paradox at a time when organs such as kidneys, ${ }^{2}$ lung, and liver ${ }^{3}$ have been transplanted from one person to another is due to the formidable difficulties of having to work under the conditions of an emergency. The resources of a large hospital and a fully experienced team must be available, and the operation may upset the routine of even a well-staffed hospital. The patient himself may have other more serious injuries that require immediate attention, while the condition of the severed limb may make it unsuitable for re-attachment-for example, it may be extensively damaged. Moreover, despite knowledge gained from surgery of war-wounds the technical procedures of anastomosis of vessels and nerves are often difficult. Complications may arise after operation : the patient may pass into a state of shock, due probably to changes in the $\mathrm{pH}$ of the blood $^{4}$; necrosis of muscle and soft tissue may develop ; and causalgia may necessitate subsequent amputation. Even if replacement is apparently successful, the limb may eventually be capable only of rather inadequate function.

Successful replacement of limbs in dogs has been undertaken on several occasions by C. C. Snyder and R. P. Knowles. ${ }^{5}$ Apparently the first successful replacement of a limb in man was done in 1962 at the Massachusetts General Hospital by R. A. Malt and C. F. McKhann, ${ }^{6}$ in a 12 -yearold boy whose right arm had been avulsed in a train 
accident, and who was admitted 30 minutes after the accident. Four months after the operation active contraction appeared in the flexor muscles of the forearm, and thereafter strength increased progressively. At 11 months 2-point discrimination, temperature, and light-touch sensations were present in all the fingers, while at 20 months the strength in the biceps, pronator teres, and opponens pollicis was about $30 \%$ to $40 \%$ of normal and in the flexors of the fingers and wrist about $80 \%$ of normal. Abduction of the shoulder was possible to $75^{\circ}$, and normal flexion of the elbow was possible, though it lacked $10^{\circ}$ full extension. The boy could lift $10 \mathrm{lb}$. $(4.5 \mathrm{~kg}$.) weights and write his name. Malt and McKhann performed another successful replacement of an arm in September, 1963, but chronologically the honour of the second case must go to C. W. Ch'en and his colleagues in Shanghai. ${ }^{7}$ Their patient, a 27-year-old man, was able to write and to play ping-pong after 7 months. Since then Ch'en and his colleagues have carried out two further successful replacements. ${ }^{8}$

The decision to carry out replacement must be assessed anew in every case. The value of the part to be replaced depends on position-thus in general the arm is progressively more valuable from shoulder to hand, but the reverse is true for the leg. A prosthesis may offer the prospects of rapid rehabilitation and a useful artificial limb. The patient must be active, with a healthy attitude towards his injury, and should not have extensive injuries elsewhere. The prognosis is better with a cleanly severed limb than with one that has been avulsed, since avulsion may stretch nerves or injure nerve roots. Without refrigeration a limb can probably survive for 4 to 6 hours, but Malt and McKhann recommend that the limb should be wrapped in aluminium foil or plastic sheeting and immersed in a mixture of ice and saline: this procedure, they believe, may increase the viability up to 18 hours. Before operation thrombus must be removed from major arteries and veins of the limb by removing obvious clots with instruments and by "milking" the limb with pressure. Perfusion of the main artery with heparinized Ringer's solution or with low-molecular-weight dextran will also help to clear the vessels of sludged and altered blood. It is essential from the first to achieve bony fixation, usually by excision of small pieces of bone and by intramedullary nailing of the main fragments. It may be necessary to shorten the bone to allow approximation of nerves and blood vessels without tension. The veins are then anastomosed, followed by the arteries ; any dead or devitalized parts of the walls of the vessels should be removed, and a graft (for example, of a saphenous vein) may be necessary to bridge the defects. Most workers in this field consider that primary suture of nerves should be undertaken. Attention should then be paid to the soft tissues, with the removal of dead tissue; skin grafting may be undertaken immediately or within a few days, the type of graft depending on the extent of the skin loss. The patient should be given tetanus prophylaxis and antibiotics. Post-operatively the limb should be splinted and elevated, and passive movement of joints should start early.

Replacement operations make considerable demands on all concerned, including the patient, who may be turned by a prolonged stay in hospital into a psychological cripple. The

1 Frost, H. M., Clin. Orthop., 1963, 29, 29.

2 Brit. med. F., 19к0, 2, 1001 .

- Ibid., 1964, 1, 514 .

- Mehl, R. L., Panl, H. A., and Beattie, E. J., jun., Lancet, 1964, $2,1419$.

S Snyder, C. C., and Knowles, R. P., Clin. Orthop., 1963, 29, 113.

- Malt, R. A., and McKhann, C. F., 尹. Amer. med." Ass., 1964, 189, 716.

' Ch'en, C. W., Ch'ien, Y. C., and Pao, Y. S., Chin. med. Ұ., 1963, 82, 632.

- Horn, J. S., Lancet, 1964, 1, 1152

- Shaw, R. S., Clin. Orthop., 1963, 29, 56. aim must be to ensure a better result by repair than would be produced by amputation and an artificial limb. Thus, even with increased knowledge and experience on the part of surgeons, the operation seems unlikely to become commonplace. As R. S. Shaw ${ }^{9}$ points out, the fascination of the possibility of accomplishing a surgical stunt must not obscure the surgeon's view of accomplishing his primary object-the maximum degree of rehabilitation for his patient.

\section{The Olympic Games}

What are the limits of human performance? Athletes at the Olympic Games soon to begin at Tokyo will probably extend some of them. But are we near the end of recordbreaking runs and jumps ? ${ }^{1}$

This seems to be a common view. In $1926 \mathrm{~A}$. V. Hill published his famous book Muscular Activity, ${ }^{2}$ incorporating the studies conducted on athletes at Princeton. A graph shows average speed in the then world records plotted against distance. It seemed unlikely then, so smooth is the resultant curve, that any substantial alteration would occur. But in fact there has been a considerable increase in speed in every event, and the improvement continues. So we must resist the temptation to say we have arrived, or nearly arrived, at the ultimate goal unless we have good scientific reasons based on the structure of the body, speed of muscle action, and other factors. At present it appears to be too difficult to make the calculations with sufficient accuracy. Nevertheless, examining the rate of change in performance provides some guide to the future, and proportional as well as absolute change appears to be greater in the long-distance and endurance events. We seem to be nearer the limit in the 100 yards than in the mile; improved performance is even greater in the long-distance events-20,000 to 30,000 metres.

Why performance continues to improve is uncertain. A number of factors probably play a part, but there is no certainty about the degree to which they contribute. Perhaps the most obvious is that the population from which athletes are drawn has greatly increased since the modern Games were first held in 1896. The number of countries taking part has increased steadily, the population of the countries has increased, and within each country there has probably been an increase in the number taking part in athletics.

Average height and weight have been increasing in those countries where measurements have been made, and the implication is that athletes in general are stronger to-day than 50. years ago. The anthropometry of the Olympic athlete was studied in detail by J. M. Tanner at the 1960 Olympic Games. ${ }^{3}$ If comparable work had been done in 1910 it would have been possible to determine whether there had been significant changes in the body size and conformation of the athlete. As a record alone, therefore, Tanner's investigation is of value. It will be possible in the future to make such comparisons. A limited study of athletes was

2 See Brit. med. 9., 1960, 2, 721.

2 Hill, A. V., Muscular Activity. Baltimore. 1926.

Tanner, J.M., The Physique of the Olympic Athlete. London. 1964.

- Kohlrausch, W., 1929, Arbeirsphysiol., 2, 187.

Krogh, A., The Axatomy and Physiology of the Capillaries. Yale University Press. 1922 .

- Hensel, H., and Hildebrandt, G., in Handbook of Physiology, Section 4: Adaptation to the Emvironment, p. 73. Washington, D.C., American
Physiological Society. 1964. 